\title{
Use of waste from the processing of Sanky pulp (Corryocactus brevistylus) to obtain a food additive.
}

Erick Alvarez-Yanamango, Eng ${ }^{1}$, Franco Vietti, $\mathrm{Bs}^{1}$, y Fredy Huayta, $\mathrm{Msc}^{1}$,

${ }^{1}$ Pontificia Universidad Católica del Perú, Grupo de Investigación en Tecnología de Procesos Agroindustriales (ITEPA), Perú, erick.alvarez@pucp.pe,ffvietti@pucp.pe, fhuayta@pucp.edu.pe

\begin{abstract}
The objective of the research was to develop a process to obtain powdered mucilage from waste or residues generated after the mechanical separation of juice or pulp from the Sanky fruit. The residue was exposed to an aqueous-alkaline extraction process, evaluating the effect of time, temperature and pH on the viscosity of the extract obtained. Subsequently, the viscous extract was dryed by atomization, using $5-10 \%$ w/v maltodextrin DE10 as encapsulant; which allowed obtaining a powder additive with rheological properties similar to gum arabic, commonly used as a stabilizer and processing aid in the food and pharmaceutical industry.

Keywords- Corryocactus brevistylus, Sanky, natural additives rheological properties, food additive.
\end{abstract}

Digital Object Identifier (DOI):http://dx.doi.org/10.18687/LACCEI2018.1.1.292

ISBN: 978-0-9993443-1-6

ISSN: 2414-6390

16 $^{\text {th }}$ LACCEI International Multi-Conference for Engineering, Education, and Technology: "Innovation in Education and Inclusion", 19-21 July 2018, Lima, Peru. 


\title{
Aprovechamiento de residuos del procesamiento de pulpa de Sanky (Corryocactus brevistylus) para la obtención de un aditivo alimentario.
}

\author{
Erick Alvarez-Yanamango, Eng ${ }^{1}$, Franco Vietti, $\mathrm{Bs}^{1}$, y Fredy Huayta, $\mathrm{Msc}^{1}$, \\ ${ }^{1}$ Pontificia Universidad Católica del Perú, Grupo de Investigación en Tecnología de Procesos Agroindustriales (ITEPA), Perú, \\ erick.alvarez@pucp.pe,ffvietti@pucp.pe,fhuayta@pucp.edu.pe
}

\begin{abstract}
The objective of the research was to develop a process to obtain powdered mucilage from waste or residues generated after the mechanical separation of juice or pulp from the Sanky fruit. The residue was exposed to an aqueous-alkaline extraction process, evaluating the effect of time, temperature and pH on the viscosity of the extract obtained. Subsequently, the viscous extract was dryed by atomization, using $5-10 \% w / v$ maltodextrin DE10 as encapsulant; which allowed obtaining a powder additive with rheological properties similar to gum arabic, commonly used as a stabilizer and processing aid in the food and pharmaceutical industry.
\end{abstract}

Keywords - Corryocactus brevistylus, Sanky, natural additives, rheological properties, food additive.

Resumen-La investigación tuvo como objetivo desarrollar un proceso para obtener mucilago en polvo a partir de las mermas o residuos que se generan tras la separación mecánica del zumo o pulpa del fruto Sanky. El residuo fue expuesto a un proceso de extracción acuosa-alcalina, evaluando el efecto del tiempo, temperatura y $\mathrm{pH}$, sobre la viscosidad del extracto acuso obtenido. Posteriormente, el extracto viscoso fue deshidratado por atomización, usando maltodextrina al 5-10 \% w/v DE10 como encapsulante; lo que permitió obtener un aditivo en polvo con propiedades reológicas similares a la goma arábiga, comúnmente usado como estabilizante y coadyuvante de procesos en la industria alimentaria y farmacéutica.

Palabras clave - Corryocactus brevistylus, Sanky, aditivos naturales, propiedades reológicas, aditivo alimentario.

\section{INTRODUCCIÓN}

El sanky (Corryocactus brevistylus) o Socconporo en quechua, es una planta que crece en las alturas de la sierra peruana, cuyos cultivos son silvestres en Ayacucho y se viene dando de forma intensiva en Huaytara-Huancavelica (2.658 msnm).

Hasta hace unos años el aprovechamiento del Sanky era limitado, por presentar dificultades en el manejo post-cosecha y el desconocimiento de su potencial alimentario, siendo su consumo de manera mínima y de manera fresca en las regiones que lo producen [Arévalo et al, 2002]. No obstante, en la actualidad es considerado un fruto promisorio, teniendo importancia económica para los pobladores de la sierra sur del Perú, principalmente Huancavelica. El fruto provee una pulpa que viene siendo estudiada por sus propiedades nutricionales y funcionales para su inclusión en el desarrollo de alimentos, lo que esta incrementado considerablemente su explotación comercial.

Su pulpa es bajo en contenido de azúcares y presenta un sabor ligeramente ácido, su contenido de potasio es superior al de frutas como el plátano hasta en un $40 \%$; posee $43.40 \mathrm{mg}$ de vitamina $\mathrm{C} / 100 \mathrm{~g}$ de pulpa, y una gran capacidad antioxidante cuantificado en $474.8 \mathrm{ug}$ eq.Trolox/g [Nolazco \& Guevara, 2007; Malaga \& Rodriguez, 2014]. Debido a algunas de estas propiedades la pulpa de Sanky ha sido incluido en el desarrollo de bebidas funcionales, ya sea de forma individual o acompañada con pulpas de otras frutas tropicales como la maracuyá o piña [Alvarez et al, 2017; Málaga \& Rodríguez, 2014; Mostacero, 2015]; incluso se ha evaluado la sustitución de la sacarosa en la formulación de las bebidas por el uso de stevia y /o sucralosa [Evangelista et al 2015, Mostacero, 2015]. Los resultados de estas iniciativas terminan siendo valiosas para la masificación del consumo del fruto y sus derivados.

El Sanky es un fruto cuyo rendimiento en pulpa es mayor al 50\%, de cuya separación se obtiene como residuos a cascaras ricas en fibra, y semillas que viene acompañadas de mucilago sumamente viscoso. El rendimiento de la separación de la semilla va de un 10 a 25\% [Nolazco \& Guevara, 2007; Málaga \& Rodriguez, 2014], y termina siendo dependiente del estado de madurez y la sobre exposición a la agitación mecánica de la pulpa; influyendo directamente sobre la viscosidad de la pulpa, aumentando la adherencia de la pulpa a la semilla y por consecuencia, aumenta el residuo durante su procesamiento [Alvarez et al, 2017].

Este fenómeno se debe principalmente al mucilago de la semilla del Sanky, que en particular es una fibra soluble que en un medio acuoso, ligeramente ácido y mediante agitación, tiende a aumentar considerablemente su volumen y mantiene una capa de humedad alrededor de la semilla, otorgándole una naturaleza viscosa al residuo.

Debido a ello, la investigación se centró en utilizar el residuo generado al separar mecánicamente las semillas de la pulpa del fruto, comúnmente denominado torta, para obtener de ella un biopolímero de naturaleza viscosa para ser utilizado como un aditivo en procesos alimentarios o farmacéuticos.

\section{MATERIALES Y MÉTODOS}




\section{A. Procesamiento del Sanky: obtención de residuos}

El Sanky (Corryocactus brevistylus), fue obtenido mediante recolección en campo a $2650 \mathrm{msnm}$ en el Distrito de Huaytara (Huancavelica, Perú), entre los meses de JunioOctubre. El procesamiento del fruto recolectado se realizó en una planta agroindustrial utilizando equipos a escala piloto, siguiendo las operaciones detalladas en la Fig. 1. De la operación de pulpeado se obtuvo una torta de naturaleza viscosa, el cual fue la materia prima para la obtención del aditivo.

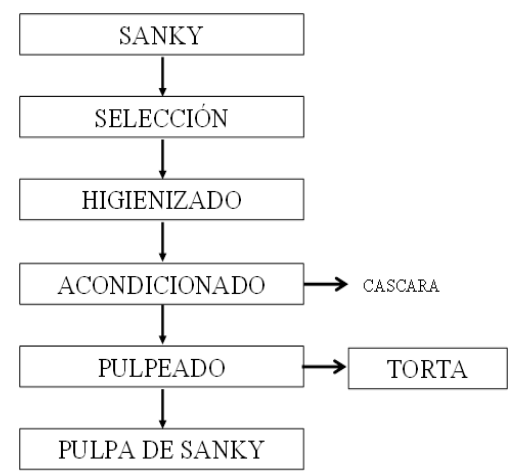

Fig. 1 Procesamiento del fruto de Sanky (Corryocactus brevistylus)

\section{B. Diseño del proceso de extracción y estabilización del aditivo obtenido a partir del residuo (torta).}

Una vez obtenida la torta como residuo, se ejecutaron las operaciones de la Fig. 2.

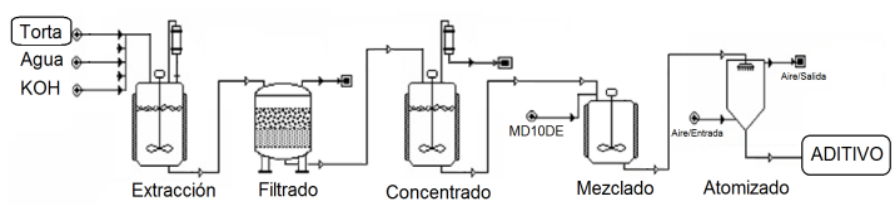

Fig. 2 Diagrama de flujo del proceso para la producción del aditivo alimentario a partir de la torta de Sanky.

Para establecer las mejores condiciones de extracción del mucilago de la torta de Sanky, se experimentó un diseño factorial completo $2^{3}$ (Tabla I), teniendo como variables de estudio al $\mathrm{pH}(8-10)$, temperatura $\left(60-80^{\circ} \mathrm{C}\right)$ y tiempo (1-2), cuyos niveles fueron establecidos mediante ensayos preliminares. Para esta fase se tuvo como variable respuesta a la viscosidad del extracto obtenido ( $\mathrm{Cp}$ máximo). Para determinar la viscosidad, se usó un Viscotester iQ HAAKE ThermoFischer (Alemania), con un spindle de cilindro concéntrico y Peltier para mantener constante la temperatura de $20^{\circ} \mathrm{C}$. Las pruebas se ejecutaron a una velocidad de corte (shear rate) de $0-1936 \mathrm{~Hz}$ por 5 minutos, obteniéndose las gráficas de comportamiento Viscosidad aparente vs. Shear rate, determinándose la máxima viscosidad para cada tratamiento.
TABLA I

DISEÑO EXPERIMENTAL DECODIFICADO PARA EL PROCESO DE EXTRACCIÓN DEL MUCILAGO CONTENIDO EN LA TORTA DE SANKY

\begin{tabular}{|c|c|c|c|}
\hline Tratamiento & $\mathrm{pH}$ & $\begin{array}{c}\text { Temperatura } \\
\left({ }^{\circ} \mathrm{C}\right)\end{array}$ & $\begin{array}{c}\text { Tiempo } \\
\text { (horas) }\end{array}$ \\
\hline 1 & 10 & 60 & 1 \\
\hline 2 & 8 & 60 & 1 \\
\hline 3 & 10 & 80 & 2 \\
\hline 4 & 10 & 80 & 1 \\
\hline 5 & 8 & 60 & 2 \\
\hline 6 & 8 & 80 & 2 \\
\hline 7 & 8 & 80 & 1 \\
\hline 8 & 10 & 60 & 2 \\
\hline
\end{tabular}

La atomización del mucílago obtenido en las condiciones óptimas ensayadas, se realizó mediante un atomizador de boquilla a presión a escala piloto. Se consideró como parámetros de operación a una temperatura de aire caliente $130 \pm 2{ }^{\circ} \mathrm{C}$, flujo de alimentación $0.98 \pm 0.02 \mathrm{~L} /$ hora $\mathrm{y}$ proporción de encapsulante (maltodextrina DE10) de 5 y $10 \%$ W/W. El rendimiento de polvos secos obtenidos (y), se determinó de acuerdo a la ecuación (1).

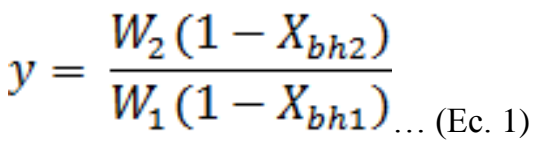

Donde, $\mathrm{W}_{1}$ es el peso del extracto de mucilago en $\mathrm{g}, \mathrm{X}_{\mathrm{bh} 1}$ es el contenido de humedad del extracto expresado en base humedad, $\mathrm{W}_{2}$ es el peso del polvo atomizado obtenido en $\mathrm{g}, \mathrm{X}_{\mathrm{bh} 2}$ es el contenido de humedad del polvo atomizado obtenido expresado en base humedad.

\section{Caracterización del aditivo obtenido.}

La caracterización del polvo comprendió el desarrollo de las pruebas físicas, químicas y reológicas.

Los ensayos físico-químicos, comprendieron la higroscopicidad, solubilidad, humedad y actividad de agua. La Higroscopicidad, se obtuvo siguiendo el procedimiento propuesto por (Cai \& Corke, 2009). Se pesó de 1- 2g del aditivo en polvo obtenido en una capsula previamente pesada; seguidamente se colocó en un recipiente con tapa hermética que contenía una solución saturada de $\mathrm{Na}_{2} \mathrm{SO}_{4}(81 \% \mathrm{HR})$, y se incubo a $25{ }^{\circ} \mathrm{C}$. Después de 7 días, se pesó la capsula y se determinó la humedad higroscópica, expresado como g de humedad por $100 \mathrm{~g}$ de sólidos secos (g / $100 \mathrm{~g})$. Para determinar la Solubilidad, se adaptó el método propuesto por Mimouni et al. (2009). Se preparó $40 \mathrm{~mL}$ una suspensión al $2 \% \mathrm{w} / \mathrm{v}$ del aditivo en polvo (5 y $10 \% \mathrm{MD}$ ), el cual fue constantemente agitado en un agitador magnético VELP Scientifica (ARE, Italia). Se pesará aproximadamente $5 \mathrm{~mL}$ de la suspensión en una placa Petri previamente pesado y se colocará a $102^{\circ} \mathrm{C}$ en una estufa Memmert (UN30, Alemania) durante $24 \mathrm{~h}$ para su posterior pesaje. La diferencia de pesos se expresará como sólidos totales (ST). Por otra parte, se centrifugará una alicuota de la suspensión a $5000 \mathrm{rpm}$ durante 30min y se separará el sobrenadante. Se pesará

16 ${ }^{\text {th }}$ LACCEI International Multi-Conference for Engineering, Education, and Technology: "Innovation in Education and Inclusion”, 19-21 July 2018, Lima, Peru. 
aproximadamente $5 \mathrm{~mL}$ del sobrenadante en una placa Petri previamente pesado y se colocará a $102^{\circ} \mathrm{C}$ en una estufa Memmert (UN30, Alemania) durante $24 \mathrm{~h}$ para su posterior pesaje. La diferencia de pesos se expresará como sólidos solubles (SS). La solubilidad (SD) o fracción másica de sólidos solubles respecto a los totales, se calculará de acuerdo a la siguiente ecuación (3):

$$
\mathrm{SD}=\mathrm{SS} / \mathrm{ST} \ldots(\text { Ec. } 3)
$$

Para determinar la Actividad de agua se usó un analizador de actividad de agua Novasina (LabSwift-aw, España). Mientras que la Humedad, se obtuvo mediante el método gravimétrico indirecto por desecación (AOAC, 2012), utilizando una estufa Memmert (UN30, Alemania) a una temperatura de $135^{\circ} \mathrm{C}$ por 2 horas.

Las pruebas reológicas practicadas al aditivo en polvo obtenido, se realizaron a suspensiones de $0.5-2 \% \mathrm{w} / \mathrm{v}, \mathrm{y}$ fueron comparadas con el comportamiento reológico de gomas comerciales $(0.5-2 \% \mathrm{w} / \mathrm{v}$ de goma arábiga y $0.1 \% \mathrm{w} / \mathrm{v}$ de goma guar). El análisis se realizó utilizando un Viscotester iQ HAAKE ThermoFischer (Alemania), a una temperatura de $20^{\circ} \mathrm{C}$ y a un shear rate de $0-1936 \mathrm{~Hz}$ por 5 minutos, obteniéndose las gráficas de comportamiento Viscosidad aparente vs. Shear rate; las cuales fueron modeladas por el modelo Power-Ostwald.

\section{RESULTADOS Y DISCUSIONES}

\subsection{Del procesamiento del Sanky: obtención de residuos}

En la Tabla II, se muestras los rendimientos promedios de la separación de la pulpa, semilla y cascara en algunas investigaciones realizadas al fruto. El rendimiento de pulpa obtenido en el estudio $(50.9 \pm 14.7)$ tiene valores similares a los reportados por Málaga \& Rodríguez (2014) y Nolazco \& Guevara (2007).

TABLA II

RENDIMIENTO DEL PROCESAMIENTO DEL FRUTO DE SANKY

\begin{tabular}{|c|c|c|c|c|}
\hline Componente & $\begin{array}{c}\text { Evangelista } \\
\& \text { Rivas, } \\
2015\end{array}$ & $\begin{array}{c}\text { Málaga \& } \\
\text { Rodríguez, } \\
2014\end{array}$ & $\begin{array}{c}\text { Nolazco \& } \\
\text { Guevara, } \\
2007\end{array}$ & $\begin{array}{c}\text { En el estudio } \\
\text { X } \pm \text { D.S }\end{array}$ \\
\hline Pulpa & 35.92 & 53.64 & 57.7 & $50.9 \pm 14.7$ \\
\hline Cascara & 40.67 & 35.42 & 35.2 & $25.4 \pm 5.0$ \\
\hline Semilla (torta) & 23.41 & 10.90 & 7.1 & $23.7 \pm 3.4$ \\
\hline
\end{tabular}

La diferencia de los reportes del rendimiento de la separación de las semillas de la pulpa entre los diferentes autores (Tabla 2), pueden deberse a factores intrínsecos del fruto, como su estado de madurez, o factores tecnológicos, como la técnica de extracción. Durante el procesamiento del Sanky, se obtuvo un $23.7 \%$ de torta, compuesta por una mezcla de pulpa y semillas, que en su conjunto presento una apariencia viscosa; resultados que difieren de los obtenidos por Málaga \& Rodríguez (2014) y Nolazco \& Guevara (2007); diferencia que puede deberse a que los referidos autores suspenden o diluyen en agua a la pulpa entera previo al pulpeado (separación mecánica). De esta manera, se facilita la separación de las semillas de la pulpa y mejora considerablemente la recuperación de la pulpa destinada a la elaboración de néctares. Por otro lado, los rendimiento de torta obtenido, concuerdan con los reportados por Evangelista \& Rivas (2015), debido a que la separación mecánica de la semillas se realizó de manera directa usando un pulpeador de características técnicas similares al usado en el presente estudio, lo que aumenta significativamente la viscosidad de la pulpa y genera una mayor cantidad de merma o torta durante la operación.

\subsection{De la extracción y estabilización del aditivo obtenido a partir del residuo.}

En la Fig. 3, se muestra la gráfica del comportamiento reológico de los extractos acuosos obtenidos de acuerdo al diseño experimental de la Tabla 1, permitiendo obtener la viscosidad máxima de cada tratamiento para el análisis estadístico.

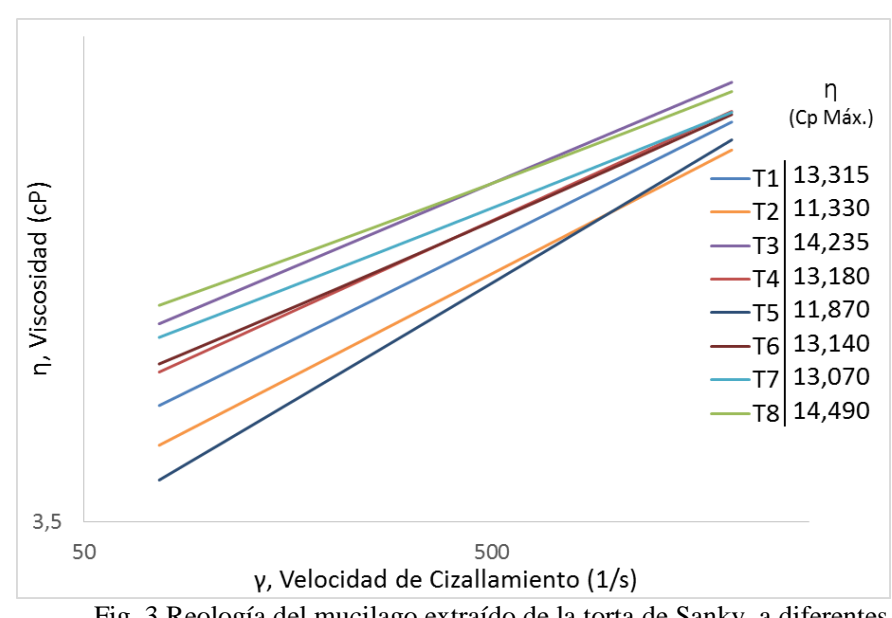

Fig. 3 Reología del mucilago extraído de la torta de Sanky, a diferentes condiciones.

Como resultado del análisis estadístico al diseño experimental, se obtuvo la Fig. 4, donde se muestra la gráfica de contornos de los efectos de los factores de estudio frente a la viscosidad, lo cual fueron obtenidas mediante el software Minitab®17. Las condiciones óptimas para cada variable que intervinieron en el proceso de extracción de mucílago de Sanky fueron: una temperatura de extracción de $60{ }^{\circ} \mathrm{C}$, un tiempo de extracción $2 \mathrm{~h}$ y a un $\mathrm{pH}$ de 10 .

A las condiciones óptimas de extracción del mucilago de Sanky se obtuvo un rendimiento del $3.74 \%$ en base seca, valores mayores a los reportados por Vargas et al. (2016) y Rodríguez (2017), quienes obtuvieron $2,56 \%$ y $1,96 \%$ respectivamente, durante la extracción acuosa y precipitación con alcohol del mucílago del nopal.

16 $^{\text {th }}$ LACCEI International Multi-Conference for Engineering, Education, and Technology: "Innovation in Education and Inclusion", 19-21 July 2018, Lima, Peru. 

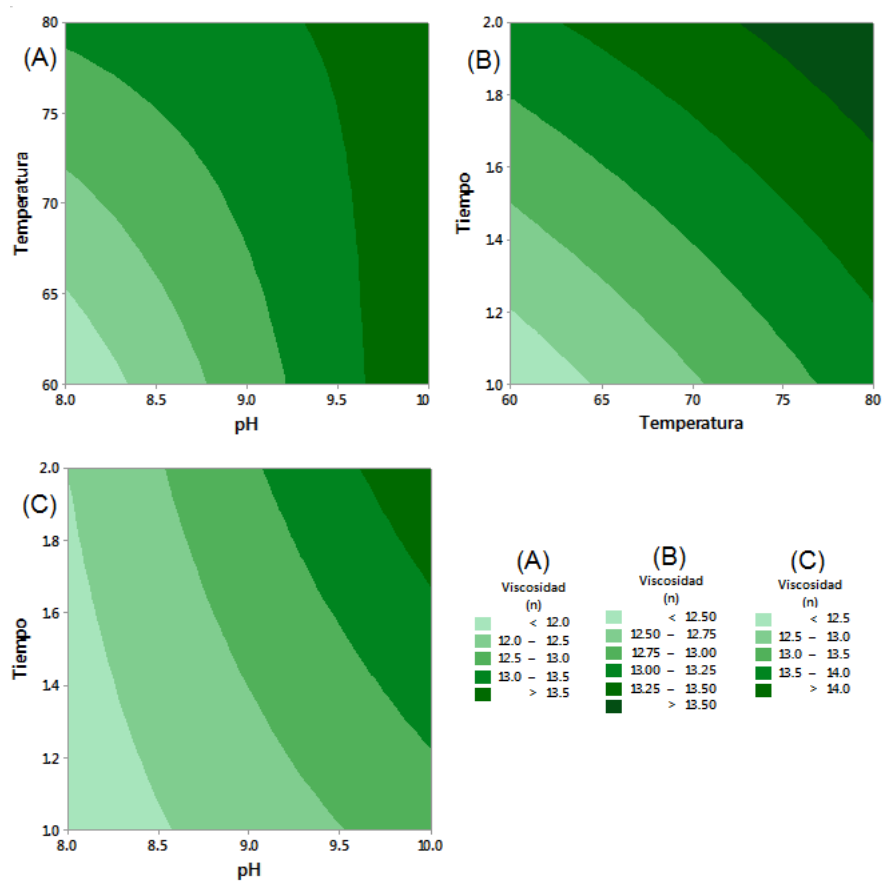

Fig. 4 Grafica de contorno de las variables de estudio en función a la viscosidad máxima del extracto acuoso.

Durante la atomización del mucilago extraído a condiciones óptimas, se obtuvo un rendimiento del $23.91 \%$ y $25.10 \%$ b.s, al usar maltodextrina al 5 y $10 \%$ como agente encapsulante.

\subsection{De la caracterización física, química y reológica del aditivo obtenido.}

En la Tabla III, se muestra los resultados de la evaluación fisicoquímica practicado al mucilago atomizado de Sanky a 2 condiciones de encapsulamiento: al 5 y $10 \%$ de Maltodextrina DE10.

TABLA III

CARACTERÍSTICAS FISICOQUÍMICAS DEL MUCILAGO ATOMIZADO DE SANKY

\begin{tabular}{|c|c|c|}
\hline Propiedad & $5 \% \mathrm{MD}$ & $10 \% \mathrm{MD}$ \\
\hline $\begin{array}{c}\text { Higroscopicidad (g agua/g } \\
\text { DM) }\end{array}$ & 0.92 & 0.73 \\
\hline Solubilidad (\%) & 95.57 & 98.02 \\
\hline Humedad (\%) & 5.98 & 4.55 \\
\hline Actividad de agua & 0.414 & 0.287 \\
\hline
\end{tabular}

En la Figura 5, se muestra el comportamiento reológico de los mucilagos atomizados de Sanky encapsulados al 5 y 10 $\%$ (b.h) de Maltodextrina DE10, considerando suspensiones a $0.5,1$ y $2 \%$ w/v.

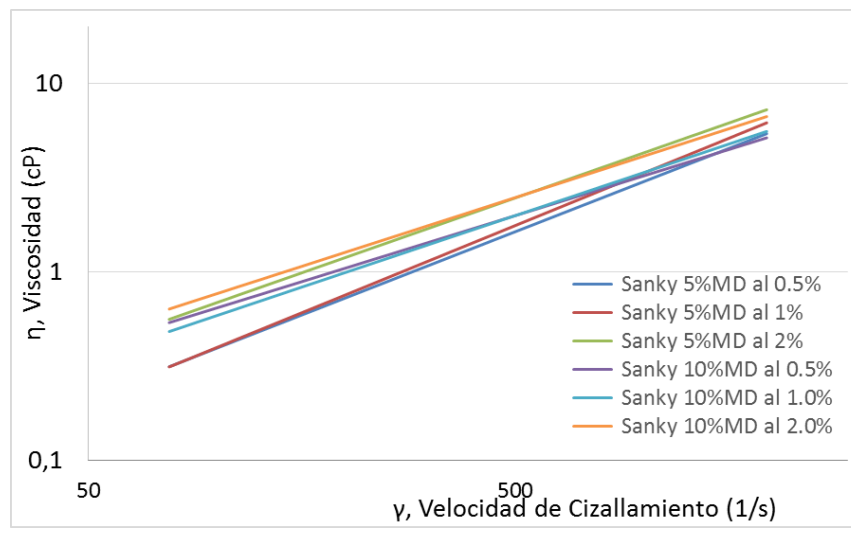

Fig. 5 Comportamiento reológico del mucilago atomizado de Sanky encapsulado con Maltodextrina.

Finalmente, en la Figura 6 se muestra el comportamiento reológico de dos gomas comerciales: goma arábiga al 0.5-2 \% w/v y goma guar al $0.1 \% \mathrm{w} / \mathrm{v}$, y su comparación con el aditivo obtenido.

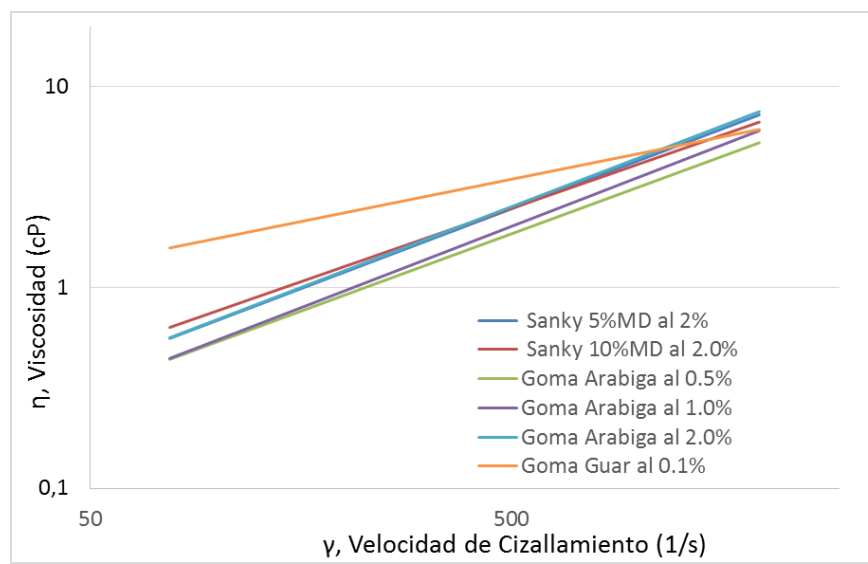

Fig. 6 Comportamiento reológico del mucilago atomizado de Sanky vs otras gomas comerciales

\section{CONCLUSIONES}

Bajo las condiciones ensayadas durante el proceso de extracción acuosa-alcalina del mucilago de Sanky, se encontró como parámetros optimizados a un $\mathrm{pH} 10$, temperatura $60^{\circ} \mathrm{C}$ y 2 horas.

Durante el atomizado del mucilago de Sanky el uso de encapsulante al 5 y $10 \%$ de Maltodextrina $10 \mathrm{DE}$, no afecto significativamente a los rendimientos del proceso.

El comportamiento reológico del mucilago en polvo obtenido de la torta del Sanky, presento resultados similares al de la goma arábiga en suspensiones del $0.5-2 \% \mathrm{w} / \mathrm{v}$; lo que viabiliza su utilización de este como un posible aditivo alimentario.

$16^{\text {th }}$ LACCEI International Multi-Conference for Engineering, Education, and Technology: "Innovation in Education and 


\section{AGRADECIMIENTOS}

Los autores agradecen al Vicerrectorado de Investigación de la Pontificia Universidad Católica del Perú por el financiamiento al Proyecto $\mathrm{N}^{\circ}$ 524, CAP 2015-PUCP.

\section{REFERENCIAS}

Arévalo A., García J., Armas V., García J., Rodríguez O., Iparraguirre R., Bartolo L., Rodríguez P., "Uso de biopectinasa y filtración al vacío para la clarificación de una mezcla diluida de pulpa de Sancayo (Corryocactus brevistylus) y tuna (Opuntia ficusindica) a diferentes temperaturas", Revista Agroindustrial Science, vol. 1, 2002.

Nolazco D. \& Guevara, "Elaboración de néctar de sanqui (Corryocactus brevistylus subsp. puquiensis)". Tesis para obtener el título profesional de industrias alimentarias. Universidad Nacional Agraria La Molina. 2007.

Málaga C. \& Rodríguez M., "Proceso para la obtención de un néctar funcional a partir de sanky (corryocactus brevistylus) maracuyá (passiflora edulis), y agua mineral procedente de yura". Tesis para obtener el título profesional de ingeniera en industrias alimentarias. Universidad Nacional de San Agustín. Arequipa - perú. 2014

Alvarez E., Vietti F., Obregón H., Atoche W., Huayta, F. (2017). Desarrollo de Néctares Hipocalóricos Mixtos con Inclusión de Frutos Nativos: Selección y Evaluación de la calidad. En 15th LACCEI International Multi-Conference for Engineering, Education, and Technology. BOCA RATON-EEUU.

Evangelista W. \& Rivas R., "Efecto de los edulcorantes (sucralosa y stevia) sobre las características sensoriales de una bebida a base de sanky (Corryocactus brevistylus)". Tesis para obtener el título profesional de ingeniero de alimentos. Universidad Nacional del Callao. Callao- Perú, 2015.

Mostacero O., "Elaboración de néctar funcional a base de sancayo o sanky (Coryucactus brevistytus) y piña (Ananá) con adición de edulcorante stevia. Tesis para optar el título de ingeniero en industria alimentaria. Universidad Catolica de Santa Maria. Arequipa Perú, 2015.

AOAC INTERNATIONAL. (2012). Official Methods of Analysis. 19th Ed.
Ibañez, M. C., \& Ferrero, C. (2003). Extraction and characterization of the hydrocolloid from Prosopis flexuosa DC seeds. Food Research International, 36(5), 455-460. http://doi.org/10.1016/S09639969(02)00192-8

Mimouni, A.; Deeth, H.; Whittaker, A.; Gidley, M.; Bhandari, B. (2009). Rehydration process of milk protein concentrate powder monitored by static light scattering. Food Hydrocolloids, 23, 1958-1965. doi:10.1016/j.foodhyd.2009.01.010

Vargas-Rodríguez, L., Arroyo Figueroa, G., Herrera Méndez, C. H., Pérez Nieto, A., García Vieyra, M. I., Rodríguez Núñez, J. R. (2016). Propiedades físicas del mucílago de nopal. Acta Universitaria, 26(NE-1), 8-11. doi: $10.15174 /$ au.2016.839

Rodríguez C., "Evaluación del mucílago de nopal (Opuntia ficus-indica) como agente estabilizante en néctar de maracuyá (Passiflora edulis)". Tesis para optar el título profesional de ingeniero de alimentos. Universidad de La Salle. Bogota - Colonbia. 2017. 